\title{
SOME COMMUTATIVITY RESULTS FOR RINGS WITH TWO-VARIABLE CONSTRAINTS
}

\author{
H. E. BELL
}

ABSTRACT. It is proved that an associative ring $R$ has nil commutator ideal if for each $x, y \in R$, there is a polynomial $p(X) \in X \mathbf{Z}[X]$ for which $x y-y p(x)$ is central. Two restrictions on the $p(X)$ which guarantee commutativity are established.

Let $\mathcal{P}$ denote the set of those polynomials in two noncommuting inde。 terminates which have integer coefficients and constant term zero. We consider associative rings $R$ with the property that for each ordered pair $(x, y)$ of elements of $R$, there exists a polynomial $p(X, Y) \in \mathcal{P}$, depending on $(x, y)$, for which

$$
x y-p(x, y) \in Z,
$$

where $Z$ denotes the center of $R$.

Putcha and Yaqub [6] have shown that if each $p(X, Y)$ in (1) is a sum of terms each of degree at least two in both $X$ and $Y$, then $R^{2} \subseteq Z$, and hence, by a long-standing theorem of Herstein [4], $R$ has nil commutator ideal. Unless the $p(X, Y)$ in (1) are restricted in some fashion, $R$ may be badly noncommutative-indeed the ring of $2 \times 2$ matrices over $G F(2)$ satisfies a condition of type (1), obtained by linearizing the identity $x^{2}=x^{8}$. However, less severe restrictions than those imposed by Putcha and Yaqub, while not implying that any power of $R$ is central, will still yield the result that $R$ has nil commutator ideal; and this note deals with one such condition, together with some special cases of it which actually yield commutativity.

Letting $X \mathbf{Z}[X]$ denote the ring of polynomials over the integers which have zero constant term, we state our major theorem as follows:

Theorem 1. Let $R$ be a ring such that for each ordered pair $(x, y)$ of elements of $R$ there exists a polynomial $p(X) \in X \mathbf{Z}[X]$, depending on $(x, y)$, for which

$$
x y^{\prime}-y p(x) \in Z \text {. }
$$

Then the commutator ideal $C(R)$ is nil and the nilpotent elements of $R$ form an ideal.

\section{Proof of Theorem 1.}

Lemma 1. Let $R$ be a ring satisfying an identity $q(X)=0$, where $q(X)$ is a polynomial in a finite number of noncommuting indeterminates, its coef-

Received by the editors June 4, 1974 and, in revised form, October 14, 1974. AMS (MOS) subject classifications (1970). Primary 16A70. 
ficients being integers with highest common factor 1 . If there exists no prime $p$ for which the ring of $2 \times 2$ matrices over $G F(p)$ satisfies $q(X)=0$, then $R$ has nil commutator ideal and the nilpotent elements of $R$ form an ideal.

The proof of this lemma, which depends on a deep result of Amitsur on PI-rings, may be found in [2].

Lemma 2. Let $R$ be a ring satisfying the hypothesis of Theorem 1 and having no nonzero divisors of zero; and let $(x, y)$ be an arbitrary ordered pair of elements of $R$. If $p(X) \in X \mathbf{Z}[X]$ is such that $x y-y p(x) \in Z$, then $x y^{2}=y^{2} x$ or $x y=y p(x)$.

Proof. Suppose that $x y^{2} \neq y^{2} x$, and write

and let $p_{1}(X) \in X \mathbf{Z}[X]$ be such that

$$
x^{2} y-y p_{1}\left(x^{2}\right) \in Z \text {. }
$$

Repeated substitution of (3) in (4) yields $x(y p(x)+z)-y p_{1}\left(x^{2}\right) \in Z$, $(y p(x)+z) p(x)+x z-y p_{1}\left(x^{2}\right) \in Z$, and finally

$$
y\left((p(x))^{2}-p_{1}\left(x^{2}\right)\right)+z(x+. p(x)) \in Z \text {. }
$$

If $(p(x))^{2}-p_{1}\left(x^{2}\right) \neq 0,(5)$ implies that $x y=y x$, contrary to our supposition that $x y^{2} \neq y^{2} x$; hence

$$
(p(x))^{2}-p_{1}\left(x^{2}\right)=0 \text { and } z(p(x)+x) \in Z,
$$

so that $z=0$ or $p(x)+x \in Z$. But if $p(x)+x \in Z$, then (3) yields $x y-y p(x)$ $=x y-y(p(x)+x)+y x \in Z$, implying that $y$ commutes with $x y+y x$ and, hence, that $y^{2}$ commutes with $x$; therefore $z=0$ and (3) now shows that $x y=y p(x)$.

Proof of Theorem 1. It will suffice to show that prime rings satisfying the hypothesis of Theorem 1 are commutative (see [2]). Accordingly, let $R$ be such a prime ring; we first show that $R$ has no nonzero divisors of zero. Suppose that $a b=0, a \neq 0$, and $r$ is an arbitrary element of $R$. There exists $q(X) \in X \mathbf{Z}[X]$ for which $b(r a)-(r a) q(b) \in Z$; and since $a q(b)=0$, we have $b(r a) \in Z$ and thus $s a(b r a)=0=(b r a) s a$ for all $s \in R$. The primeness of $R$ now implies that $b r a=0$ and hence that $b=0$.

Assume that $R$ is a noncommutative prime ring satisfying (2). The identity

$$
\left(x y^{2}-y^{2} x\right)\left(y x^{2}-x^{2} y\right)\left(x y^{2} x-y x^{2} y\right)=0
$$

is not satisfied by the ring of $2 \times 2$ matrices over any field $G F(p)$, as may be verified by substituting the matrices $\left[\begin{array}{ll}1 & 1 \\ 1 & 0\end{array}\right]$ and $\left[\begin{array}{ll}1 & 0 \\ 0 & 0\end{array}\right]$ for $x$ and $y$ respectively; thus, by Lemma $1, R$ cannot satisfy (7), and there must exist elements $a, b$ of $R$ for which $a b^{2}-b^{2} a, b a^{2}-a^{2} b$, and $a b^{2} a-b a^{2} b$ are all nonzero. 
If $p(X) \in X \mathbf{Z}[X]$ is such that $a b-b p(a) \in Z$, it follows from Lemma 2 that

$$
a b=b p(a) .
$$

Now let $s(X) \in X \mathbf{Z}[X]$ satisfy

$$
b p(a)-p(a) s(b) \in Z
$$

and apply the result of Lemma 2 to the ordered pair $(b, p(a))$. If $(p(a))^{2} b=$ $b(p(a))^{2}$, it follows from (8) that $a^{2} \cdot b=a(b p(a))=b(p(a))^{2}=(p(a))^{2} b$, so that $a^{2}=(p(a))^{2}$ and $a^{2}$ commutes with $b$, contrary to the choice of $a$ and $b$. Therefore, by Lemma $2, b p(a)=p(a) s(b)$, which combines with (8) to give

$$
a b=p(a)_{s}(b) \text {. }
$$

Now it is immediate from Lemma 2 that $R$ is an Ore domain and can be embedded in a division ring $D$. In $D,(10)$ implies that $b(s(b))^{-1}=a^{-1} p(a)$ commutes with both $a$ and $b$; and (8) written in the form $a b=b a a^{-1} p(a)$ shows that $a b$ and $b a$ commute, contrary to the original choice of $a$ and $b$. This contradiction completes the proof of Theorem 1 .

2. Two commutativity theorems. In this section we single out two conditions of type (2) which imply commutativity.

Theorem 2. Let $R$ be a ring such that for every ordered pair $(x, y)$ of elements of $R$, there exists an integer $n=n(x, y) \geq 1$ for which $x y=y x^{n}$. Then $R$ is commutative.

Lemma 3. Any ring $R$ satisfying the hypothesis of Theorem 2 has each of the following properties:

(a) Idempotents of $R$ are central.

(b) $R$ is a duo ring (i.e. one-sided ideals are two sided); moreover $a b=0$ implies $b a=0$, so that there is no distinction between right and left zero divisors.

(c) Commutators in $R$ are central.

(d) If $a, b \in R$ are such that $a(a b-b a)=b(a b-b a)=0$, then $a b-b a=$ 0 ; similarly, if $a(a b-b a) x=b(a b-b a) x=0$ for some $x \in R$, then $(a b-b a) x=0$.

Proof. (a) If $x \in R$ and $e$ is idempotent, there exist positive integers $m, n$ such that $e(e x-e x e)=(e x-e x e) e^{m}$ and $e(x e-e x e)=(x e-e x e) e^{n}$; hence $e x-e x e=x e-e x e=0$.

(b) Let $I$ be a right ideal of $R, a \in I$ and $r \in R$; note that since $r a=a r^{n}$ for some $n \geq 1, r a \in I$. Thus all right ideals are two-sided, and a similar argument holds for left ideals.

Now let $a b=0$. Since $b a=a b^{n}$ for some $n \geq 1, b a=0$ as well.

(c) By Theorem 1 the commutator ideal is nil and, hence, contained in the Jacobson radical $J(R)$; therefore, it will suffice to show $J(R) \subseteq Z$. If we 
assume the existence of an element $a \in J(R) \backslash Z$, then there is an element $b \in R$ and integers $m, n>1$ for which $a b=b a^{m}$ and $b a=a b^{n} \neq a b$. It follows that $a b=a b b^{n-1} a^{m-1}$; and because $b^{n-1} a^{m-1} \in J(R)$, we now have $a b=0$. Similarly, $b a=0$ and we have a contradiction.

(d) Suppose $a(a b-b a)=b(a b-b a)=0$; in view of (c), $a^{2} b=b a^{2}$ and $b^{2} a=a b^{2}$. Suppose $a b-b a \neq 0$ and let $m, n>1$ be such that $a b=b a^{m}$ and $b a=a b^{n}$. Substituting each of these expressions into the other yields $a b=$ $a b^{n} a^{m-1}$ and $b a=b a^{m} b^{n-1}$. If $m$ and $n$ are both even we thus get $a b=$ $b a=a^{m} b^{n}$; on the other hand, if one of $n, m$ is odd, we have

$$
a b-b a=a b b^{n-1} a^{m-1}-b a a^{m-1} b^{n-1}=(a b-b a) a^{m-1} b^{n-1},
$$

which is zero since $(a b-b a) a=0$.

Finally, if $x \in R$ and $A$ is the annihilator of $x$, we get the second statement of (d) by applying the preceding argument to the ring $R / A$.

Proof of Theorem 2. It will suffice to prove commutativity under the additional hypothesis that $R$ is subdirectly irreducible, in which case (since $R$ is a duo ring) the set of zero divisors is precisely the annihilator of the unique minimal ideal $S$ [1, Lemma 3].

The initial step is to show that zero divisors in $R$ are central. Accord. ingly, suppose $a$ is a noncentral zero divisor which fails to commute with some element $b \in R$; and consider the case where $b$ is also a zero divisor. Then by (d) of Lemma 2, we have one of $(a b-b a) a$ and $(a b-b a) b$ different from 0 and $(a b-b a) R$ is a nontrivial ideal; therefore if $0 \neq s \in S$, there exists an element $x \in R$ for which $s=(a b-b a) x$. But $0=a s=b s=$ $a(a b-b a) x=b(a b-b a) x$, and from (d) of Lemma 2 we then get $(a b-b a) x=0$, a contradiction. Now consider the case where $b$ is not a zero divisor and let $m, n>1$ be such that $a b=b a^{m}$ and $b a=a b^{n}$. Since $a b$ is a zero divisor, $a b$ and $a$ commute, so that $a(a b-b a)=(a b-b a) a=0$ and $a^{2}$ commutes with $b$. If $m$ is odd, repeating some of the computation in Lemma 2(d) shows that $a b-b a=(a b-b a) a^{m-1} b^{n-1}=0$; on the other hand, if $m$ is even, $a b=a^{m} b, a^{m}=a$, and $a^{m-1}$ is a nonzero idempotent. Recalling that any nonzero central idempotent of a subdirectly irreducible ring must be a multiplicative identity element, we get a contradjction of the fact that $a$ was a zero divisor. Therefore zero divisors of $R$ are central.

Now suppose that $R$ is not commutative and $b \notin Z$. There then exist $a \in R$ not commuting with $b$ and an integer $j>1$ such that $b a=a b^{j}$. Since $a$ cannot be a zero divisor and since $a b-b a=a\left(b-b^{j}\right)$ is a zero divisor (nilpotent, in fact), $b-b^{j}$ must be a zero divisor, hence central. We have now arrived at a contradiction of Herstein's well-known result that a ring $R$ is commutative if for each $x \in R$, there is an integer $n(x)>1$ for which $x-x^{n(x)} \in Z$; and our proof is complete.

Theorem 3. Let $R$ be a ring such that for every ordered pair $(x, y)$ of 
elements of $R$, there is a polynomial $p(X) \in X \mathbf{Z}[X]$ such that $x y=y x p(x)$. Then $R$ is commutative.

Proof. Again applying the given condition to $e, e x-e x e$, and $x e-e x e$ shows that idempotents must be central. Also, since $x^{2}=x^{2} p(x)$ for some $p(X) \in X \mathbf{Z}[X], R$ is periodic by a result of Chacron [3]; therefore, $R$ is either nil or contains a nonzero idempotent.

Suppose now that $R$ is subdirectly irreducible. If $R$ contains a nonzero idempotent, then it must have an identity; thus, for each $x \in R$ we have $x=$ $x p(x)$, and $R$ is commutative by the major theorem of [5]. On the other hand, if $R$ is nil we have

$$
x y=y x p(x)=x y q(y) p(x)=y x p(x) q(y) p(x)=y x q(y) r(x)
$$

for an appropriate element $r(X) \in X \mathbf{Z}[X]$. In particular, $x y=y x y z_{1}$ for some element $z_{1} \in R$; and, continuing inductively, for each positive integer $n$ we get an element $z_{n} \in R$ for which $x y=y^{n} x y z_{n}$, so that $x y=0$ and $R$ is a zero ring. Therefore, if $R$ is subdirectly irreducible, it is commutative; and the proof of Theorem 3 is finished.

The hypothesis of Theorem 3 cannot be weakened to the condition that $x y-y x p(x) \in Z$, as we see by noting that there exist noncommutative rings satisfying the identity $x^{2}=0$. However, it may be of some interest (but not enough to justify including the proof) to note that rings satisfying the weaker hypothesis are polynomial-identity rings-satisfying the identity $[[x, y], z]^{2}[x, y]=0$.

\section{REFERENCES}

1. H. E. Bell, Duo rings: some applications to commutativity theorems, Canad. Math. Bull. 11 (1968), 375-380. MR 38 \#3297.

2. - On some commutativity theorems of Herstein, Arch. Math. (Basel) 24 (1973), 34-38. MR 47 \#8631.

3. M. Chacron, On a theorem of Herstein, Canad. J. Math. 21 (1969), 13481353. MR $41 \# 6905$.

4. I. N. Herstein, A theorem on rings, Canad. J. Math. 5 (1953), 238-241. MR $14,719$.

5. - The structure of a certain class of rings, Amer. J. Math. 75 (1953), 864-871. MR 15, 392.

6. M. S. Putcha and A. Yaqub, Rings satisfying polynomial constraints, J. Math. Soc. Japan 25 (1973), 115-124. MR 47 \#1867. CANADA

DEPARTMENT OF MATHEMATICS, BROCK UNIVERSITY, ST. CATHARINES, ONTARIO, 